\title{
The Functional Properties of Barrel Cortex Neurons Projecting to the Primary Motor Cortex
}

\author{
Takashi R. Sato and Karel Svoboda \\ Janelia Farm Research Campus, Howard Hughes Medical Institute, Ashburn, Virginia 20147
}

Nearby neurons, sharing the same locations within the mouse whisker map, can have dramatically distinct response properties. To understand the significance of this diversity, we studied the relationship between the responses of individual neurons and their projection targets in the mouse barrel cortex. Neurons projecting to primary motor cortex (MI) or secondary somatosensory area (SII) were labeled with red fluorescent protein (RFP) using retrograde viral infection. We used in vivo two-photon $\mathrm{Ca}^{2+}$ imaging to map the responses of RFP-positive and neighboring L2/3 neurons to whisker deflections. Neurons projecting to MI displayed larger receptive fields compared with other neurons, including those projecting to SII. Our findings support the view that intermingled neurons in primary sensory areas send specific stimulus features to different parts of the brain.

\section{Introduction}

In the mouse barrel cortex (Woolsey and van der Loos, 1970), the locations of the neurons within the somatotopic map account for only a small part of the response heterogeneity; even neighboring neurons sharing the same barrel column can have very different whisker selectivities (Sato et al., 2007). Does this heterogeneity reflect highly specific subcolumnar circuits (Song et al., 2005; Yoshimura et al., 2005) or simply noise in neocortical wiring? The heterogeneity of neural responses could be related to the diverse cell types intermingled within the cortex, such as excitatory and inhibitory cells (Simons, 1978; Brumberg et al., 1999; Sohya et al., 2007). Neurons with specific projection targets can also have distinct response properties (Evarts, 1965; Hikosaka and Wurtz, 1983; Swadlow, 1988, 1989; Movshon and Newsome, 1996; Ferraina et al., 2002; Briggs and Usrey, 2009). Projectiondependent neuronal function has been probed with extracellular unit recordings combined with antidromic activation (Evarts, 1965; Movshon and Newsome, 1996; Ferraina et al., 2002), but this method is inefficient, even between areas with strong connections [e.g., V1 and MT in the macaque (Movshon and Newsome, 1996)]. In addition, this method does not provide information on the precise locations of the antidromically stimulated neurons, and projection-dependent functional differences could thus still be related to cell location (e.g., cortical layer or minicolumn) (Ferraina et al., 2002) rather than the projection pattern per se.

To overcome these limitations, we combined bulk loading of a green Ca indicator (Stosiek et al., 2003) and retrograde labeling

Received May 26, 2009; revised Nov. 27, 2009; accepted Dec. 16, 2009.

We thank Susana Lima, Tony Zador, and Ed Callaway for information related to retrograde viruses, Patrick Kanold, Takaki Komiyama, Na Ji, and Mac Hooks for extensive discussions, Noah Gray, Chris Harvey, Mariko Izumo, Alla Karpova, and Tianyi Mao for advice on molecular cloning, Asaf Keller, Kevin Alloway, Ford Ebner, and Dan Simons for advice on anatomy and microstimulation, and Takaki Komiyama, Dan O'Connor, and Leopoldo Petreanu for comments on the manuscript.

Correspondence should be addressed to Takashi R. Sato, Janelia Farm Research Campus, Howard Hughes Medical Institute, 19700 Helix Drive, Ashburn, VA 20147. E-mail: satot@janelia.hhmi.org.

D0I:10.1523/JNEUROSCI.3774-09.2010

Copyright $\odot 2010$ the authors $\quad 0270-6474 / 10 / 304256-05 \$ 15.00 / 0$ with a virus [herpes simplex virus 1 (HSV1)] (Lilley et al., 2001) expressing a red fluorescent protein (RFP; DsRed-Express) (see Fig. $1 A, B)$. This method allows us to simultaneously image the responses of retrogradely labeled (RFP + ) and nearby nonlabeled $(\mathrm{RFP}-)$ neurons. We found that neurons that project to the primary motor cortex (MI) have larger receptive fields compared with other neurons, including those that project to secondary somatosensory area (SII).

\section{Materials and Methods}

Virus preparation. cDNA of DsRed-Express was subcloned between EcoRI and BsrGI in the HSV1 shuttle vector obtained from Biovex. Expression of DsRed-Express was driven by the EF1 $\alpha$ promoter. A disabled version of HSV1 was produced by Biovex (Lilley et al., 2001; Lima et al., 2009).

Virus injection. All experimental protocols were conducted according to the National Institutes of Health guidelines for animal research and were approved by the Institutional Animal Care and Use Committee at Janelia Farm Research Campus. C57BL/6J mice (Charles River) were deeply anesthetized using an isoflurane-oxygen mixture ( $1 \%$ vol isoflurane/vol $\mathrm{O}_{2}$ ) at postnatal day $12-14$ and placed in a custom stereotactic apparatus. A small incision was made in the skin to identify the location of bregma, and then was immediately closed and sutured. Then, another small incision was made and small holes were drilled in the scalp at the injection site, either MI (1.2 $\mathrm{mm}$ anterior to bregma, $0.6 \mathrm{~mm}$ lateral) or SII ( $0.7 \mathrm{~mm}$ posterior to bregma, $4.2 \mathrm{~mm}$ lateral). Microstimulation in MI resulted in whisker movements. Fifty nanoliters of viral suspension (titer, $2 \times 10^{9} \mathrm{pfu} / \mathrm{ml}$ ) was injected (over $1 \mathrm{~min}$ ) using a pulled glass micropipette (tip diameter, $10-20 \mu \mathrm{m}$; Drummond). To prevent backflow, the micropipette was left in the brain for $10 \mathrm{~min}$ before pulling up. The scalp was closed and sutured, and then the animals were allowed to recover on a heated pad. RFP expression took place for 7-14 $\mathrm{d}$ before the imaging experiments. Similar procedures were used for bead injections (undiluted stock, LumaFluor).

Animals and surgical procedures. Mice (postnatal day 20-27) were anesthetized using an isoflurane-oxygen mixture ( $1 \%$ vol isoflurane/vol $\mathrm{O}_{2}$ ). Core body temperature was maintained at $37^{\circ} \mathrm{C}$ using a heating blanket (Harvard Apparatus). Imaging windows were installed above the barrel cortex. A small craniotomy (diameter, $1-2 \mathrm{~mm}$ ) was made $1 \mathrm{~mm}$ 
posterior from bregma and $3.5 \mathrm{~mm}$ lateral from the midline on the right hemisphere. The intact dura was covered with $2 \%$ agarose (Type-IIIA, Sigma), dissolved in HEPES-buffered artificial CSF, and a $5 \mathrm{~mm}$ cover glass (World Precision Instruments). The cover glass was sealed in place using dental acrylic, leaving one side open for electrode access (Svoboda et al., 1997, 1999; Sato et al., 2007).

Loading procedures. Following the craniotomy, the mice were placed under a custom-made two-photon microscope and anesthetized with ketamine $(160 \mathrm{mg} / \mathrm{kg})$ and xylazine $(10 \mathrm{mg} / \mathrm{kg})$. Layer $2 / 3$ neurons in the barrel cortex (rows D, C, B; arcs 1, 2, 3, 4, 5) were loaded with Fluo-4 AM (F14201, Invitrogen) using multi-cell bolus loading (Stosiek et al., 2003; Ohki et al., 2005; Sato et al., 2007). The choice of Fluo-4 AM, compared with other calcium indicators (e.g., Oregon Green Bapta-1 AM), was based on its high signal-to-noise ratio (Sato et al., 2007). Fluo-4 AM was dissolved in 20\% (w/v) Pluronic F-127 (P-6867, Invitrogen) in DMSO to a concentration of $10 \mathrm{~mm}$. This solution was then diluted 10 -fold into external buffer containing (in $\mathrm{mm}$ ): $125 \mathrm{NaCl}, 5 \mathrm{KCl}, 10$ glucose, 10 HEPES, $2 \mathrm{CaCl}_{2}$, and $2 \mathrm{MgSO}_{4}$. In some experiments, 1-100 nм Alexa was added to visualize the flow. Glass micropipettes (tip resistance, $\sim 1$ $\mathrm{M} \Omega$ ) were filled with the loading solution and inserted into the barrel cortex. The tips of the micropipettes were brought into a cortical region expressing DsRed-Express. Repetitive pulses of positive pressure (5-10 psi, 10 ms, 10-20 times; PicoSpritzer II, General Valve) were applied to eject the dye to bulk load a small tissue volume (diameter, $\sim 200 \mu \mathrm{m}$ ).

Two-photon microscopy. In vivo imaging was performed using a custom-made two-photon laser-scanning microscope controlled by ScanImage software (Pologruto et al., 2003). The light source for Fluo-4 imaging was a pulsed Ti:sapphire laser $(\lambda, \sim 810 \mathrm{~nm} ; 50-150 \mathrm{~mW}$ in the objective back-focal plane; MaiTai, Spectra-Physics) and for DsRedExpress imaging was a solid state Ytterbium laser $(\lambda, \sim 1030 \mathrm{~nm} ; 100-$ $200 \mathrm{~mW}$ in the objective back-focal plane; t-Pulse, Amplitude Systemes). Red and green fluorescence photons were separated using a $565 \mathrm{~nm}$ dichroic mirror (Chroma Technology) and barrier filters (green, BG22; red, 607/45; Chroma Technology). Signals were collected using photomultiplier tubes (3896 or H7422P-40 MOD, Hamamatsu). The objective lens (40, 0.8 NA) and trinoc were from Olympus. We used frame scanning (frame rate, $15.6 \mathrm{~Hz}$ ) with $2 \mathrm{~ms}$ line durations $(32 \times 1024$ pixels). Pixel dimensions were $0.10 \times 1.3 \mu \mathrm{m}$. Images were collected in layer 2 and 3, 120-250 $\mu \mathrm{m}$ from the top of the dura (Woolsey and van der Loos, 1970; Bureau et al., 2006; Sato et al., 2007).

Sensory stimulation. The loading pipette was used to measure the sensory stimulation-evoked local field potential (LFP). A hand-held stimulator was used to identify whiskers that were effective in evoking LFPs in the area loaded with $\mathrm{Ca}^{2+}$ indicator. These whiskers were then deflected using a piezoelectric stimulator $(300 \mu \mathrm{m}$ deflection in the rostral-caudal direction, positioned $5 \mathrm{~mm}$ from the base of the whisker) while recording the LFP. The pair of whiskers evoking the largest LFP response [principal whisker $(\mathrm{PW})$ ] and the second largest LFP response [surround whisker (SW)] were used for further mapping.

Imaging sessions began $30 \mathrm{~min}$ after dye loading. Trial durations were $960 \mathrm{~ms}$ (15 frames). To minimize use-dependent depression, intertrial intervals were long (20-30 s) (Armstrong-James, 1975) (supplemental Fig. 2, available at www.jneurosci.org as supplemental material). Whiskers were stimulated in an interleaved manner. The imaging session lasted 1-1.5 h (100-200 trials). Two factors conspired against longer imaging sessions. First, Fluo-4AM was extruded from the neuronal cytoplasm. Second, extracellular baseline fluorescence increased with time due to unknown causes (Koester et al., 1999).

In some experiments, after the imaging sessions fluorescent beads were injected into the center of the imaging site, and the PW was verified anatomically by examining the barrel pattern in tangential sections (100 $\mu \mathrm{m}$ thick) through layer 4 stained with cytochrome oxidase (Wong-Riley and Welt, 1980; Land and Simons, 1985).

Data analysis. Single whisker deflections cause zero, one, or, rarely, two action potentials in L2/3 neurons (Simons, 1978; Armstrong-James and Fox, 1987; Svoboda et al., 1997; Brecht and Sakmann, 2002; Brecht et al., 2003; Sato et al., 2007). Action potentials cause $\mathrm{Ca}^{2+}$ accumulations in somata and dendrites that can be detected with $\mathrm{Ca}^{2+}$ imaging in vivo (Svoboda et al., 1997, 1999; Waters and Helmchen, 2004). Because indi-

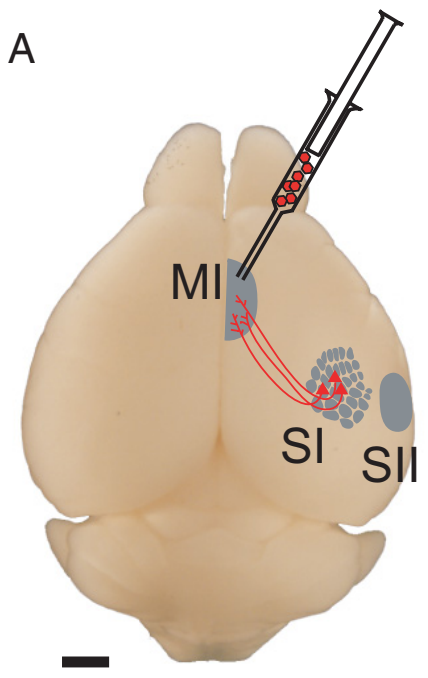

$1 \mathrm{~mm}$

C

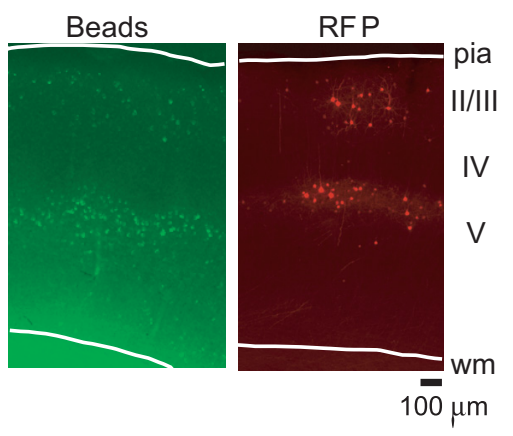

B

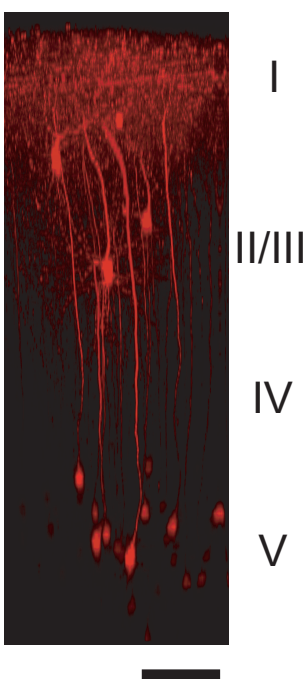

$100 \mu \mathrm{m}$

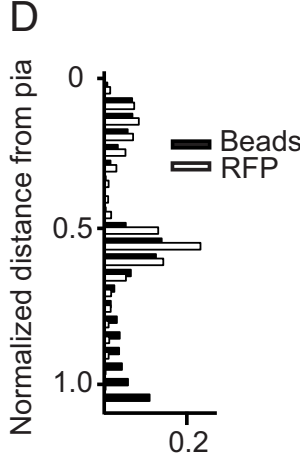

Fraction of cells
Figure 1. Retrograde labeling with a virus expressing a red fluorescent protein. $A$, Schematic showing retrograde labeling of neurons in SI by injection of the retrograde virus HSV1 into MI. $\boldsymbol{B}$, In vivo image of RFP + neurons (maximum-intensity side projection of an image stack of RFP + neurons; $512 \times 128 \times 96$; section spacing, $8 \mu \mathrm{m}$ ). C, Distribution of labeled neurons in SI barrel cortex after bead (left) or virus (right) injection into MI. The white lines indicate the pia and the border between the cortex and the white matter. $\boldsymbol{D}$, Normalized distribution of labeled neurons after bead (black, 1293 neurons) and HSV (white, 808 neurons) injection into MI.

vidual dendrites were difficult to identify, we performed fluorescence measurements in somata. Regions of interest were manually selected inside the somata.

Trials that resulted in action potential-evoked fluorescence changes and trials that did not produce fluorescence changes were separated using two parameters, as described previously (Sato et al., 2007): (1) The change in $\Delta F / F$ between the last prestimulus frame and the first poststimulus frame. Because spikes can occur up to $\sim 50 \mathrm{~ms}$ after whisker deflection (Glazewski et al., 1996; Brecht et al., 2003), we also computed the difference between the first and second poststimulus frames. $F_{\mathrm{d}}$ was defined as the larger of these values. (2) The amplitude, $A_{\mathrm{F}}$, of the fluorescence transient derived from template matching (Clements and Bekkers, 1997). The template was:

$$
\begin{aligned}
& \text { template }(t)=\text { offset } \quad(t \leq 0) \\
& \text { template }(t)=\text { offset }+\mathrm{AF} \cdot e^{-t / \tau}(t>0) .
\end{aligned}
$$

The time constant of the template, $\tau$, was the decay time of the exponential fluorescence transients $(1 \mathrm{~s})$. The origin $(0 \mathrm{~ms})$ of the template window ( -192 to $256 \mathrm{~ms}$, eight frames) was positioned at the frame of interest, and amplitude and offset were adjusted to fit the data by minimizing the sum of squared errors. 
We plotted $F_{\mathrm{d}}$ and $A_{\mathrm{F}}$ for all trials (see Fig. $2 B$ ) and then applied hierarchical clustering, using the Euclidean distance, to define the distance between points. For many cells, the trials fell into two groups, corresponding to failures and successes. To quantify the separation between these groups, we calculated the $95 \%$ confidence ellipse for each of the two clusters. We focused our analysis on those cells in which the two confidence ellipses were separated, and discarded the rest. Assigning responsive trials and nonresponsive trials in this manner resulted in error rates of $<1 \%$ (Sato et al., 2007).

For all neurons in which more than five responsive trials were detected, the response probability $\left(P_{\mathrm{r}}\right)$ was determined for each cell and whisker (PW, SW) as the number of responsive trials divided by the total number of trials. The ratio of these two values was calculated as follows:

$$
R=\frac{\min \left(P_{\mathrm{r}}(\mathrm{PW}), P_{\mathrm{r}}(\mathrm{SW})\right)}{\max \left(P_{\mathrm{r}}(\mathrm{PW}), P_{\mathrm{r}}(\mathrm{SW})\right)} .
$$

\section{Results}

Following injection of viral suspension into MI, RFP + neurons were found in the contralateral primary motor cortex, ipsilateral SII, ipsilateral posterior cingulate cortex (29c), ipsilateral thalamus, and layers $2 / 3$ and 5 of the ipsilateral barrel cortex. This pattern was largely consistent with previous studies using HRP injections (Porter and White, 1983) and our own data obtained with fluorescent beads, a classic retrograde tracer (Katz et al., 1984) (Fig. 1C,D). The labeling efficiency with HSV1 was lower compared with beads ( $\sim 30 \%$; comparing areas with the densest labels) (Fig. 1C, compare left and right). However, HSV1 infection provided brighter labeling, allowing us to image $\mathrm{RFP}+$ cells in vivo (Fig. $1 B$ ). In contrast, bead-labeled neurons were harder to detect in vivo.

We loaded a cluster (diameter, $\sim 200$ $\mu \mathrm{m})$ of layer $2 / 3$ neurons, including $\mathrm{RFP}+$ neurons, in the barrel cortex with the Ca indicator Fluo-4 AM (see Materials and Methods) (Fig. 2A) (Stosiek et al., 2003; Ohki et al., 2005; Sato et al., 2007). In the green fluorescence channel, loaded neurons could be identified as areas of relatively homogenous, dim green fluorescence against a highly heterogeneous fluorescent background (Fig. $2 \mathrm{~A}$, left). In the red channel, RFP+ cells showed bright red fluorescence on a dark background (Fig. $2 A$, right, cell 1 ). We used field potential measurements to determine the PW and the SW producing the largest responses, at the center of the imaging window (supplemental Fig. $1 A$, available at www.jneurosci.org as supplemental material) (Sato et al., 2007).

F
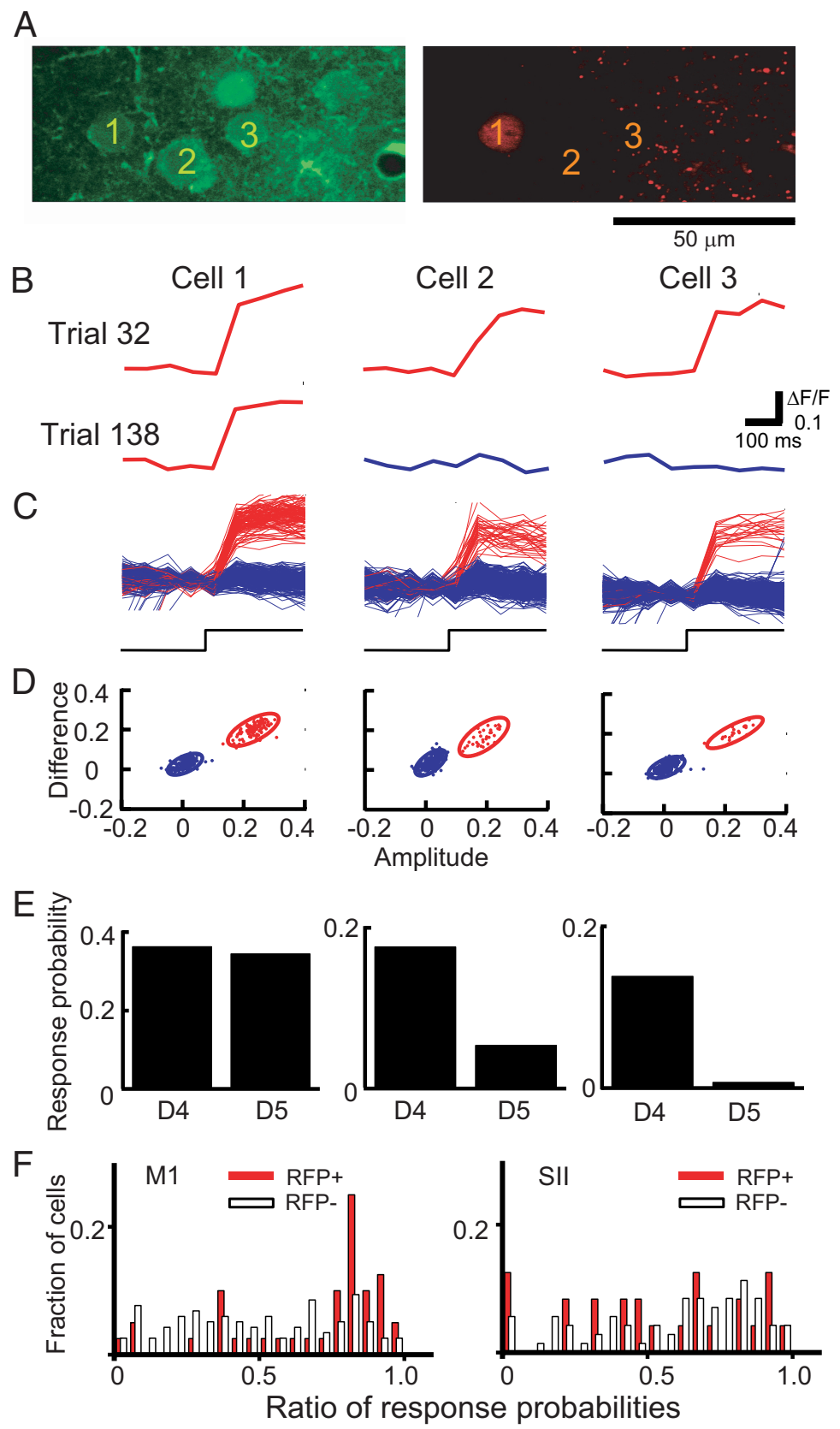

Figure 2. The receptive fields of barrel cortex neurons with projections to MI or SII. A, Left, Cells were loaded with Fluo-4 AM; right, one cell in the field of view (RFP+, cell 1) sends axons to MI. $B$, Examples of whisker stimulation-evoked fluorescence transients for three cells on two different trials. Columns correspond to the three cells identified in $\boldsymbol{A}$. Red traces indicate trials with action potential-evoked fluorescence transients, blue traces indicate trials without fluorescence transients. Whisker stimuli are indicated at the bottom of $\boldsymbol{C}$. C, Multiple fluorescence transients from cells $1-3$ aligned on the whisker stimulus (bottom). D, Plots of amplitude $\left(A_{\mathrm{F}}\right)$ and difference $\left(F_{\mathrm{d}}\right)$ (see supplemental Methods, available at www.jneurosci.org as supplemental material) for all trials for the cells identified in $\boldsymbol{A}$. The points were clustered into two groups (red, successes; blue, failures). The $95 \%$ confidence ellipses are overlaid on the graph. $\boldsymbol{E}$, The response probability of these three cells to stimulation of D4 and D5. $\boldsymbol{F}$, The distribution of the ratios of response probabilities to PW and SW stimulation following the virus injection into MI (left) and SII (right). The red bars indicate data from RFP + cells and the white bars indicate the data from RFP - cells.

We next mapped the receptive fields of Fluo-4 AM-labeled neurons by stimulating the PW and SW. Following a whisker deflection, most neurons exhibited fluorescence changes in some trials but not in other trials. In many neurons, trials with and without fluorescence changes could be reliably distinguished (e.g., cells 1-3) (Fig. 2B-D), and we focused our analysis on these neurons (256/351 cells); the remainder of the neurons, for which 
"spike sorting" was not possible, were not analyzed further. Trials with fluorescence changes correspond to trials with single action potentials, whereas trials without fluorescent changes correspond to trials without action potentials (Sato et al., 2007).

For example, the RFP + cell 1 responded equally often to stimulation of PW and SW: the response probability was 0.36 for PW stimulation (149 trials), and 0.35 for SW stimulation ( 150 trials) (Fig. 2E). However, the neighboring RFP- neurons (cells 2, 3) were more selective to PW stimulation (cell 1: PW, 0.17; SW, 0.05; cell 2: PW, 0.13; SW, 0.01). Thus, in this experiment the $\mathrm{RFP}+$ cell had a larger receptive field and a higher response probability compared with the neighboring neurons.

We quantified the receptive field size by calculating the ratio of the response probability to PW and SW stimulation. When neurons were labeled by injecting HSV1 into MI, the ratio was larger for RFP + neurons compared with RFP - neurons (RFP+ neurons, $n=40,0.71 \pm 0.26$; RFP $-, n=117,0.52 \pm 0.28$; mean $\pm \mathrm{SD} ; p<0.00001$, for comparison between the actual data and the data shuffled within each imaging session) (Fig. $2 F$, left), implying that MI-projecting neurons have larger receptive fields. In addition, the response probability for the PW was larger for $\mathrm{RFP}+$ neurons compared with RFP - neurons (RFP+ neurons, $n=40,0.61 \pm 0.24 ; \mathrm{RFP}-$ neurons, $n=120,0.55 \pm 0.27$; mean $\pm \mathrm{SD} ; p<0.05$, for comparison between the actual data and the data shuffled within each imaging session).

We next examined the functional properties of neurons projecting to SII (supplemental Fig. $1 B$, available at www.jneurosci. org as supplemental material). Using double injection of red and green fluorescent beads into MI and SII, we confirmed that only a small portion of neurons project to both MI and SII: only 40/ 993 cells were double labeled (394 cells labeled from MI, 639 cells from SII from four mice; analysis was focused on areas where the labeling was most dense) (supplemental Fig. $1 C$, available at www.jneurosci.org as supplemental material) (Chakrabarti and Alloway, 2006). Following HSV1 injection into SII, we measured the response properties of RFP + neurons in the barrel cortex (Fig. $2 F$, right). The ratio of the response probabilities to $\mathrm{PW}$ and SW stimulation were similar between RFP + and RFP - neurons (RFP + neurons, $n=24,0.57 \pm 0.31$; RFP - neurons, $n=71$, $0.62 \pm 0.27$; mean $\pm \mathrm{SD} ; p>0.50)$. The response probability was also similar (RFP + neurons, $n=24,0.58 \pm 0.27$; RFP - neurons, $n=72,0.59 \pm 0.25$; mean $\pm \mathrm{SD} ; p>0.50)$. Finally, we compared the receptive field size and the response probabilities between MI-projecting neurons and SII-projecting neurons, measured in different animals. The ratio of the response probabilities to PW and SW stimulation was larger for MI-projecting neurons compared with SII-projecting neurons $(p<0.03)$. For the response probability to PW stimulation, the difference was not significant $(p>0.10)$. Importantly, for the RFP- neurons neither the receptive field size nor the response probability to PW stimulation were significantly different between the two sets of experiments (receptive field size, $p>0.80$; response probability, $p>0.80$ ).

\section{Discussion}

Primary sensory cortices send axons to multiple cortical and subcortical areas that process different aspect of sensory information (Felleman and Van Essen, 1991; Kaas et al., 1999; Alloway, 2008). Our results are consistent with the view that different targets receive distinct types of information from the same area (Movshon and Newsome, 1996; Morishima and Kawaguchi, 2006; Brown and Hestrin, 2009). We speculate that MI receives signals related to voluntary whisking, which might not require information about individual whiskers. In contrast, SII may be involved in object recognition and require information relating touch and whisker identity (Alloway, 2008).

Our study is the first demonstration of in vivo $\mathrm{Ca}$ imaging combined with retrograde tracing. This approach has advantages over extracellular recording combined with antidromic stimulation: it allows us to compare the activity of neighboring neurons simultaneously. Thus, the functional difference can be unambiguously assigned to projection patterns rather than differences in location (Ferraina et al., 2002) or the state of animals. In contrast, antidromic stimulation methods provide information about precise spike timing and can be applied to deep brain structures (Hikosaka and Wurtz, 1983).

Understanding the logic underlying the diversity of neural responses in the cortex is an essential step in revealing cortical computation. The technique used in the current study, along with related approaches (Sohya et al., 2007; Lima et al., 2009), will be useful in assigning other types of sensory responses to particular cell types defined by their axonal projection patterns.

\section{References}

Alloway KD (2008) Information processing streams in rodent barrel cortex: The differential functions of barrel and septal circuits. Cereb Cortex 18:979-989.

Armstrong-James M (1975) The functional status and columnar organization of single cells responding to cutaneous stimulation in neonatal rat somatosensory cortex S1. J Physiol 246:501-538.

Armstrong-James M, Fox K (1987) Spatiotemporal convergence and divergence in the rat S1 "barrel” cortex. J Comp Neurol 263:265-281.

Brecht M, Sakmann B (2002) Dynamic representation of whisker deflection by synaptic potentials in spiny stellate and pyramidal cells in the barrels and septa of layer 4 rat somatosensory cortex. J Physiol 543:49-70.

Brecht M, Roth A, Sakmann B (2003) Dynamic receptive fields of reconstructed pyramidal cells in layers 3 and 2 of rat somatosensory barrel cortex. J Physiol 553:243-265.

Briggs F, Usrey WM (2009) Parallel processing in the corticogeniculate pathway of the macaque monkey. Neuron 62:135-146.

Brown SP, Hestrin S (2009) Intracortical circuits of pyramidal neurons reflect their long-range axonal targets. Nature 457:1133-1136.

Brumberg JC, Pinto DJ, Simons DJ (1999) Cortical columnar processing in the rat whisker-to-barrel system. J Neurophysiol 82:1808-1817.

Bureau I, von Saint Paul F, Svoboda K (2006) Interdigitated paralemniscal and lemniscal pathways in the mouse barrel cortex. PLoS Biol 4:e382.

Chakrabarti S, Alloway KD (2006) Differential origin of projections from SI barrel cortex to the whisker representations in SII and MI. J Comp Neurol 498:624-636.

Clements JD, Bekkers JM (1997) Detection of spontaneous synaptic events with an optimally scaled template. Biophys J 73:220-229.

Evarts EV (1965) Relation of discharge frequency to conduction velocity in pyramidal tract neurons. J Neurophysiol 28:216-228.

Felleman DJ, Van Essen DC (1991) Distributed hierarchical processing in the primate cerebral cortex. Cereb Cortex 1:1-47.

Ferraina S, Paré M, Wurtz RH (2002) Comparison of cortico-cortical and cortico-collicular signals for the generation of saccadic eye movements. J Neurophysiol 87:845-858.

Glazewski S, Chen CM, Silva A, Fox K (1996) Requirement for a-CaMKII in experience-dependent plasticity of the barrel cortex. Science 272:421-423.

Hikosaka O, Wurtz RH (1983) Visual and oculomotor functions of monkey substantia nigra pars reticulata. IV. Relation of substantia nigra to superior colliculus. J Neurophysiol 49:1285-1301.

Kaas JH, Hackett TA, Tramo MJ (1999) Auditory processing in primate cerebral cortex. Curr Opin Neurobiol 9:164-170.

Katz LC, Burkhalter A, Dreyer WJ (1984) Fluorescent latex microspheres as a retrograde neuronal marker for in vivo and in vitro studies of visual cortex. Nature 310:498-500.

Koester HJ, Baur D, Uhl R, Hell SW (1999) Ca(2+) fluorescence imaging with pico- and femtosecond two-photon excitation: signal and photodamage. Biophys J 77:2226-2236.

Land PW, Simons DJ (1985) Cytochrome oxidase staining in the rat SmI barrel cortex. J Comp Neurol 238:225-235. 
Lilley CE, Groutsi F, Han Z, Palmer JA, Anderson PN, Latchman DS, Coffin RS (2001) Multiple immediate-early gene-deficient herpes simplex virus vectors allowing efficient gene delivery to neurons in culture and widespread gene delivery to the central nervous system in vivo. J Virol 75:4343-4356.

Lima SQ, Hromádka T, Znamenskiy P, Zador AM (2009) PINP: a new method of tagging neuronal populations for identification during in vivo electrophysiological recording. PLoS One 4:e6099.

Morishima M, Kawaguchi Y (2006) Recurrent connection patterns of corticostriatal pyramidal cells in frontal cortex. J Neurosci 26:4394-4405.

Movshon JA, Newsome WT (1996) Visual response properties of striate cortical neurons projecting to area MT in macaque monkeys. J Neurosci 16:7733-7741.

Ohki K, Chung S, Ch'ng YH, Kara P, Reid RC (2005) Functional imaging with cellular resolution reveals precise micro-architecture in visual cortex. Nature 433:597-603.

Pologruto TA, Sabatini BL, Svoboda K (2003) ScanImage: flexible software for operating laser-scanning microscopes. BioMedical Engineering OnLine 2:13.

Porter LL, White EL (1983) Afferent and efferent pathways of the vibrissal region of primary motor cortex in the mouse. J Comp Neurol 214: 279-289.

Sato TR, Gray NW, Mainen ZF, Svoboda K (2007) The functional microarchitecture of the mouse barrel cortex. PLoS Biol 5:e189.

Simons DJ (1978) Response properties of vibrissa units in rat SI somatosensory neocortex. J Neurophysiol 41:798-820.

Sohya K, Kameyama K, Yanagawa Y, Obata K, Tsumoto T (2007) GABAergic neurons are less selective to stimulus orientation than excitatory neurons in layer II/III of visual cortex, as revealed by in vivo functional $\mathrm{Ca}^{2+}$ imaging in transgenic mice. J Neurosci 27:2145-2149.

Song S, Sjöström PJ, Reigl M, Nelson S, Chklovskii DB (2005) Highly nonrandom features of synaptic connectivity in local cortical circuits. PLoS Biol 3:e68.

Stosiek C, Garaschuk O, Holthoff K, Konnerth A (2003) In vivo two-photon calcium imaging of neuronal networks. Proc Natl Acad Sci U S A 100:7319-7324.

Svoboda K, Denk W, Kleinfeld D, Tank DW (1997) In vivo dendritic calcium dynamics in neocortical pyramidal neurons. Nature 385:161-165.

Svoboda K, Helmchen F, Denk W, Tank DW (1999) The spread of dendritic excitation in layer $2 / 3$ pyramidal neurons in rat barrel cortex in vivo. Nat Neurosci 2:65-73.

Swadlow HA (1988) Efferent neurons and suspected interneurons in binocular visual cortex of the awake rabbit: receptive fields and binocular properties. J Neurophysiol 59:1162-1187.

Swadlow HA (1989) Efferent neurons and suspected interneurons in S-1 vibrissa cortex of the awake rabbit: receptive fields and axonal properties. J Neurophysiol 62:288-308.

Waters J, Helmchen F (2004) Boosting of action potential backpropagation by neocortical network activity in vivo. J Neurosci 24:11127-11136.

Wong-Riley MT, Welt C (1980) Histochemical changes in cytochrome oxidase of cortical barrels after vibrissal removal in neonatal and adult mice. Proc Natl Acad Sci U S A 77:2333-2337.

Woolsey TA, Van der Loos H (1970) The structural organization of layer IV in the somatosensory region (S1) of mouse cerebral cortex. Brain Res 17:205-242.

Yoshimura Y, Dantzker JL, Callaway EM (2005) Excitatory cortical neurons form fine-scale functional networks. Nature 433:868-873. 